\title{
NGAL, Albumin and Cystatin C During Cisplatin Therapy
}

\author{
Blanka FLOROVA ${ }^{1,2}$, Daniel RAJDL ${ }^{1}$, Jaroslav RACEK ${ }^{1,2}$, Ondrej FIALA ${ }^{3}$, Vit Martin \\ MATEJKA ${ }^{3}$, Ladislav TREFIL ${ }^{1,2}$
}

${ }^{1}$ Department of Clinical Biochemistry and Hematology, Faculty of Medicine in Pilsen, Charles University and University Hospital in Pilsen, Czech Republic, ${ }^{2}$ Biomedical Center, Faculty of Medicine in Pilsen, Charles University, Czech Republic, ${ }^{3}$ Department of Oncology and Radiotherapy, Faculty of Medicine in Pilsen, Charles University and University Hospital in Pilsen, Czech Republic

Received May 20, 2019

Accepted December 13, 2019

Epub Ahead of Print March 23, 2020

\begin{abstract}
Summary
Cisplatin is a commonly used chemotherapeutic drug. It is known for its nephrotoxic side effects with an increased risk of acute kidney injury. Finding of clinically feasible cisplatin nephrotoxicity markers is of importance. In our study, we compared neutrophil gelatinase-associated lipocalin (NGAL) in serum and urine, the estimated glomerular filtration rate (based on serum cystatin $\mathrm{C}$ ) and urine albumin as markers of nephrotoxicity. The study involved 11 men and 9 women (mean \pm SD age $58.2 \pm 9.5$ years) with different malignancies treated with cisplatin in four cycles of chemotherapy (I - IV). Samples 0-4 were taken before, immediately after, in 3, 6 and 24 hours after administering chemotherapy. We detected significant increase of ACR in Sample $2(p=0.03)$ and decrease of eGFR in Sample $4(p=0.03)$ up to 24 hours after cisplatin administration in the first chemotherapy cycle only. When cumulative effect of cisplatin was assessed, significantly increased values of urine albumin (vs cycle I) were found in Sample $0 \quad(p=0.00058), 1 \quad(p=0.00256)$, $2(p=0.00456), 3(p=0.00006)$ and $4(p=0.00319)$ in cycles II to IV. We found a correlation between values of urine NGAL and urine albumin $(r=0.68, p<0.0001)$. In conclusion, urine albumin was the only measured marker that consistently and statistically significantly increased after cisplatin containing chemotherapy cycles.
\end{abstract}

\section{Key words}

Urine and serum NGAL • Urine albumin - Serum cystatin C • Nephrotoxicity • Cisplatin

\section{Corresponding author}

Daniel Rajdl, Department of Clinical Biochemistry and
Hematology, Faculty of Medicine in Pilsen, Charles University and University Hospital in Pilsen, Alej Svobody 80, 30460 Pilsen, Czech Republic. E-mail: rajdı@fnplzen.cz

\section{Introduction}

Chemotherapy is often accompanied by damage to vitally important organs during tumor treatment. After oncology therapy, patients may have many long-term side effects associated with toxic damage to organs caused by chemotherapy (e. g. heart failure or kidney damage). More effective antitumor therapy results in higher-risk survivors with a clinical manifestation of chronic damage to vitally important organs. Cisplatin ranks among so called alkylating cytostatics and is currently one of the most common chemotherapeutics (Maghsoudi et al. 2015). Use of cisplatin is limited by side effects on the body. These are especially neurotoxicity and nephrotoxicity accompanied by higher risk of acute kidney injury (AKI). AKI induced by cisplatin appears in approximately ten days after administering chemotherapy and is accompanied by decrease in glomerular filtration (eGFR), hypomagnesemia and hypocalcemia (Maghsoudi et al. 2015). Renal toxicity caused by cisplatin originates from uptake and activation of platinum in proximal tubule cells (Miller et al. 2010) and is at least partly mediated by poly(ADP-ribose) polymerase (Kim 2016).

Nowadays, increased serum creatinine and decreased urine production are primarily used in diagnosis of AKI, which are factors indicating loss of excretory renal function. Afterwards, AKI is classified 
according to RIFLE (Risk, Injury, Failure, Loss of Kidney Function, End-Stage Kidney Disease) or AKIN criteria (Acute Kidney Injury Network) (Haase et al. 2011) or recently developed KDIGO (Kidney Disease Improving Global Outcomes) classification. Since use of serum creatinine is neither sensitive nor specific, particularly in early phases of AKI, there are attempts to find a marker which would predict AKI earlier and in a more specific way (Maghsoudi et al. 2015). The mere measurement of plasmatic creatinine does not enable us to distinguish normal renal function, AKI and CKD from a temporary azotemia with dehydration (Haase-Fielitz et al. 2014). The estimation of glomerular filtration based on serum creatinine suffers mainly from influences caused by changes in muscle mass. Patients with progressive tumors are commonly malnourished and have decreased muscle mass. Serum creatinine concentrations reflect the amount of muscle mass and are lower in patients with malnutrition. And thus, the estimation of glomerular filtration can be overrated (Drott et al. 1988).

Cystatin $\mathrm{C}$ is a microprotein relatively constantly produced in all nucleated cells, freely filtered in the glomerulus and completely reabsorbed and degraded in proximal tubulus. This characteristic allows us to use cystatin $\mathrm{C}$ as a marker of glomerular filtration rate. It has proven its clinical superiority over creatinine in cases where eGFR derived from creatinine is biased (e.g. low or high muscle mass), in confirmation of decreased eGFR for diagnosis of chronic kidney disease (CKD; Kidney Disease: Improving Global Outcomes (KDIGO) CKD Work Group 2013) and as a strong predictor of mortality in patients with CKD (Xu et al. 2015) and as a predictor of AKI (Gaygisiz et al. 2016). On the other hand, cystatin $\mathrm{C}$ is a strongly regulated molecule whose expression is also modified in inflammatory and tumorous states. A significant correlation between increased serum cystatin $\mathrm{C}$ and the malignity progression of melanoma and colorectal carcinoma shows possible non-renal factors on serum cystatin $\mathrm{C}$ values in malignant states (Kos et al. 1998).

Urinary albumin is considered the best routinely available laboratory marker of kidney injury. It reflects especially injury of the glomerulus and albuminuria is important in pathophysiology of tubulointersticial damage in CKD. To reflect changing concentration in urine depending on fluid intake, ratio of albumin and creatinine is used (ACR). ACR is used in the KDIGO classification scheme to determine the prognosis of $\mathrm{CKD}$ and prediction of AKI (Deng et al. 2017). Moreover, it is used in diabetic patients as a marker of diabetic nephropathy but increased urinary albumin levels are considered as a general sign of endothelial dysfunction and are incorporated in algorithms estimating cardiovascular risk (Heerspink et al. 2015).

Neutrophil gelatinase-associated lipocalin (NGAL), or also siderocalin or lipocalin 2, appeared to be one of auspicious markers for detecting AKI. NGAL is a glycoprotein $(24 \mathrm{kDa})$ belonging to the lipocalin family (Chakraborty et al. 2012). Human NGAL was first isolated from secondary granules of human neutrophils (Devarajan, 2010) NGAL synthesis is strongly induced by ischemic and toxic insults. An increase in urine NGAL arises within two hours after kidney injury and 24-72 hours before an increase in serum creatinine (Haase et al. 2011; Mishra et al. 2003). An increase in urine NGAL correlates with the toxin dose and renal ischemia duration (Mishra et al. 2003). In one study, more than ten times higher increase in serum NGAL levels and more than hundred-time increase in urine NGAL were found in adult patients with AKI (it was defined as doubling of serum creatinine within less than five days) in comparison with the control group of patients (Nickolas et al. 2008).

Nevertheless, NGAL has its limits as a marker of AKI. Plasma NGAL measurements may be influenced by pathological states such as chronic kidney damage, chronic hypertension, systemic infection, anemia, hypoxia or malignancies. In some cases, urine NGAL measurements are not specific for detection of kidney damage and there is no consensus on the cut-off value which should be used for diagnosis of AKI (Tsigou et al. 2013).

The aim of our study was to select the best marker of cisplatin nephrotoxicity in patients undergoing chemotherapy from the following 5 markers: serum and urine NGAL, serum creatinine and cystatin $\mathrm{C}$ or urine albumin. The secondary goal was to describe time-course of these biomarkers in the setting of real-life chemotherapy cycles.

\section{Methods}

The group consisted of 20 patients (11 men and 9 women) aged between 34 and 78 (mean age \pm SD was $58.2 \pm 9.5$ years) who underwent chemotherapy protocol containing cisplatin. Most patients were administered cisdichlorodiammineplatinum (cissDDP) in concomitance with radiotherapy $(n=11)$, the second most common protocol was a combination of cissDDP and 5-fluorouracil $(n=7)$ or other cytostatics $(n=2)$. Regarding the concomitance with radiotherapy, the dose of cissDDP was $50 \mathrm{mg} / \mathrm{m}^{2}$ per week. In case of combined protocols, 
patients were administered $100 \mathrm{mg} / \mathrm{m}^{2}$ per $3-4$ weeks. Before the cisDDP infusion (one litre of normal saline with cisDPP for 2 hours), one litre of normal saline for 2 hours was infused followed by $500 \mathrm{ml}$ of normal saline and $200 \mathrm{ml}$ of mannitol.

Patients were diagnosed different types of tumours, namely nasopharyngeal carcinoma $(n=1)$, lower gingiva $(n=1)$, tongue edge $(n=2)$, tongue root $(n=1)$, glottis $(n=1)$, tonsils $(n=1)$, cardia $(n=1)$, esophageal chest $(n=2)$, urinary bladder $(n=1)$, undescended testes $(n=1)$, vagina $(n=2)$ and cervix $(n=6)$. Patients were monitored for 3 to 47 months, 4.7 months on average. During this period $(9 / 2012$ to $7 / 2016)$ eleven patients of the group died.

The aim of the treatment plan was to administer at least five chemotherapy cycles in concomitance as well as in case of palliative therapy in combination with 5-fluorouracil. The main reasons for the unfinished treatment plan was a necessary change of the platinum derivate due to decreased renal functions (nephrotoxicity; $\mathrm{n}=11$ ) or the finished or suspended protocol for hematologic toxicity $(n=5)$. Six patients refused to continue with the study due to the complicated study protocol. The clinical diagnosis of decreased renal function (nephrotoxicity) was based on declined estimated glomerular filtration rate under approx. $1 \mathrm{ml} / \mathrm{s}$. Individual patients were administered one to five cycles (2.3 cycles on average). Due to a very small number of patients in the fifth cycle $(n=2)$, we only evaluated data of the first four cycles.

This study was approved by the local ethics committee and all the patients provided informed consent.

Urine and plasma were taken in each cycle before chemotherapy (Sample 0), immediately after administering cisplatin (Sample 1), in three hours (Sample 2), in six hours (Sample 3) and in 24 hours (Sample 4) after administering cisplatin.

Serum and urine creatinine (S_crea, U_crea, resp.) concentration, serum and urine NGAL concentration, urine albumin and serum cystatin $\mathrm{C}$ were determined. Afterwards we calculated NGAL/creatinine ratio, albumin/creatinine ratio (ACR). Glomerular filtration rate was estimated by using the CKD-EPI (Chronic Kidney Disease Epidemiology Collaboration) equation for cystatin $\mathrm{C}$ (Kidney Disease: Improving Global Outcomes (KDIGO) CKD Work Group 2013).

Serum and urine creatinine concentrations were determined by using the Jaffe method (Crea, Beckman Coulter; automated biochemical analyzer AU640, Beckman Coulter). Serum cystatin C level was determined by quantitative immunoturbidimetric assay (Cystatin C AssayKit, Diazyme, supplier LabMark; automated biochemical analyzer AU640, Beckman Coulter). Urine and serum NGAL concentrations were measured by quantitative immunoturbidimetric assay (The NGAL Test ReagentKit, BioPorto, supplier LabMark; automated immunochemistry analyzer Architect $i$ 2000SR, Abbott). Urine albumin concentration was determined by quantitative immunoturbidimetric assay (Tina-quant Albumin, Roche Diagnostics; automated biochemical analyzer Cobas 6000, Roche).

We used the programs of R 3.2.0 and MedCalc 17.7.2 to evaluate the data statistically. Correlation analysis was made by using Spearman's correlation coefficient. We applied the nonparametric KruskalWallis test to compare changes among chemotherapy cycles and the Conover test of pairwise comparison of subgroups. The Jonckheere-Terpstra test was used to detect trends of medians in time. All measured markers were used as survival predictors in univariate survival analysis (Cox proportional hazard analysis) where $\mathrm{p}$ value was derived from logrank test for comparison of survival curves between patients with and without nephrotoxicity. If not stated otherwise, data are presented as medians (interquartile range). $\mathrm{P}<0.05$ was considered statistically significant.

\section{Results}

To detect acute nephrotoxic effects of cisplatin we compared changes of measured markers in the samples 0 to 4 for each individual chemotherapy cycle. We only noticed statistically significant changes in the first chemotherapy cycle, which was an increase in ACR in Sample 2 (3 hours after cisplatin administration, $\mathrm{p}=0.03$, Fig. 1A) and a decrease in eGFR in Sample 4 (24 hours after cisplatin administration, $p=0.03$, Fig. $3 A$ ). Surprisingly, a decrease in serum NGAL was borderline statistically significant in Sample 2 and Sample 3 $(p=0.045)$ in the first chemotherapy cycle. More details can be found in Table 1 .

To detect the cumulative effect of cisplatin, we compared changes in markers from Cycle I to IV in Samples 0 to 4. E.g. comparing Sample 0 values in Cycle I with Sample 0 values in Cycles II, III and IV. An increase in urine albumin and ACR was statistically significant in Cycle II, Cycle III and Cycle IV (in comparison with Cycle I, in most samples; $\mathrm{p}<0.05$, see Fig. $1 \mathrm{~A}$ and Table 1). Urine NGAL levels showed statistically significant increasing trend from Cycle I to 
Cycle IV ( $\mathrm{p}=0.03)$ in Sample 0 only. Serum and urine NGAL (Fig. 2A) and estimated glomerular filtration rate from cystatin C (Fig. 3A) evinced no statistically significant trend in medians from Cycle I to Cycle IV and the levels were not substantially different in individual cycles.

Table 1. Changes in measured marker values between individual chemotherapy cycles. Following data are expressed as median (interquartile range). In columns, aggregated values form chemotherapy Cycles I to IV are showed. Rows in each marker represent Samples 0 to 4 .

\begin{tabular}{|c|c|c|c|c|}
\hline & I. $(n=20)$ & II. $(n=11)$ & III. $(n=7)$ & IV. $(n=5)$ \\
\hline$U \_N G A L$ & $0: 43.5(34.0-100)$ & 0: $95.0(59.0-184)$ & $0: 67.0(43.3-127)$ & $0: 116(74.0-400)$ \\
\hline \multirow[t]{4}{*}{$(\mu g / l)$} & $1: 41.0(30.5-65.5)$ & $1: 67.0(44.0-96.3)$ & $1: 30.0(27.5-79.5)$ & 1: $72.0(42.8-349)$ \\
\hline & $2: 35.5(23.5-66.5)$ & 2: $51.0(29.8-103)$ & $2: 42.0(14.0-65.8)$ & $2: 70.0(35.3-352)$ \\
\hline & $3: 48.0(22.5-55.0)$ & $3: 45.0(31.5-93.8)$ & $3: 51.0(40.5-63.3)$ & $3: 54.0(40.5-151)$ \\
\hline & $4: 54.0(32.5-126)$ & $4: 81.0(43.0-150)$ & $4: 47.0(36.0-187)$ & $4: 69.0(31.0-140)$ \\
\hline$U \_N G A L / U \_$crea & $0: 8.14(4.94-21.2)$ & $0: 13.1(5.43-19.8)$ & $0: 5.68(3.26-44.9)$ & $0: 12.1(5.04-26.6)$ \\
\hline \multirow[t]{4}{*}{$(\mathrm{mg} / \mathrm{mol})$} & $1: 9.60(7.01-19.6)$ & $1: 12.3(6.99-26.9)$ & $1: 14.3(4.96-41.9)$ & $1: 12.8(3.33-61.1)$ \\
\hline & $2: 16.1(7.21-41.0)$ & 2: $17.2(8.00-28.2)$ & $2: 5.51(5.00-25.4)$ & $2: 29.2(9.54-123)$ \\
\hline & $3: 14.2(6.71-22.5)$ & 3: 13.7 (6.03-29.0) & $3: 13.3(7.35-25.8)$ & $3: 22.6(7.17-55.2)$ \\
\hline & 4: $9.16(4.04-16.3)$ & 4: $9.50(3.70-19.1)$ & 4: $10.0(4.09-20.3)$ & $4: 6.16(4.57-27.1)$ \\
\hline$U_{-} A l b$ & $0: 5.00(2.99-16.0)$ & $0: 56.0(17.0-116)^{*}$ & $0: 16.0(10.8-188)^{*}$ & $0: 98.0(39.6-406)^{*}$ \\
\hline \multirow[t]{4}{*}{$(m g / l)$} & $1: 4.50(2.99-10.0)$ & 1: $41.0(14.3-62.3)^{*}$ & $1: 8.00(3.49-188)$ & 1: $59.0(31.3-328)^{*}$ \\
\hline & $2: 2.99(2.99-7.50)$ & 2: $18.0(3.99-51.0)^{*}$ & $2: 14.0(6.00-70.0)$ & $2: 23.0(14.3-199)^{*}$ \\
\hline & $3: 2.99(2.99-4.00)$ & $3: 26.0(8.25-42.8)^{*}$ & 3: $15.0(5.00-55.3)^{*}$ & $3: 26.0(7.76-90.0)^{*}$ \\
\hline & 4: $6.00(2.99-11.5)$ & 4: $58.0(19.0-66.5)^{*}$ & 4: $38.0(10.3-88.3)^{*}$ & 4: $45.0(16.8-133)^{*}$ \\
\hline$A C R$ & $0: 1.06(0.63-3.41)$ & $0: 4.30(2.25-9.68)^{*}$ & $0: 6.19(1.44-10.4)^{*}$ & $0: 3.77(2.68-31.8)^{*}$ \\
\hline \multirow[t]{4}{*}{$(\mathrm{g} / \mathrm{mol})$} & $1: 1.57(0.84-3.41)$ & $1: 4.02(2.05-13.5)^{*}$ & $1: 6.49(1.27-11.9)$ & 1: $10.6(3.49-33.9)^{*}$ \\
\hline & $2: 2.40(1.17-4.87)^{1}$ & $2: 4.42(1.63-19.0)$ & $2: 7.04(1.72-9.34)$ & $2: 12.3(4.57-37.0)$ \\
\hline & $3: 1.46(0.86-2.24)$ & $3: 4.73(2.41-20.4)^{*}$ & $3: 6.52(1.67-8.85)^{*}$ & $3: 4.67(3.87-22.47)^{*}$ \\
\hline & 4: $0.97(0.58-1.72)$ & $4: 3.65(1.54-11.9)^{*}$ & 4: $5.35(1.94-8.27)^{*}$ & $4: 4.02(2.24-24.9)^{*}$ \\
\hline eGFR (cystatin C) & $0: 1.65(1.20-1.90)$ & $0: 1.70(1.35-1.78)$ & $0: 1.60(1.40-1.78)$ & $0: 1.50(1.30-1.76)$ \\
\hline \multirow[t]{4}{*}{$(\mathrm{ml} / \mathrm{s})$} & $1: 1.70(1.30-1.90)$ & $1: 1.70(1.43-1.80)$ & $1: 1.70(1.60-1.88)$ & $1: 1.80(1.58-2.00)$ \\
\hline & $2: 1.80(1.45-2.05)$ & $2: 1.80(1.43-1.90)$ & $2: 1.80(1.63-1.88)$ & 2: $1.80(1.43-1.96)$ \\
\hline & $3: 1.75(1.35-1.95)$ & $3: 1.80(1.20-1.88)$ & $3: 1.80(1.45-1.90)$ & $3: 1.60(1.45-2.03)$ \\
\hline & $4: 1.45(0.95-1.70)^{2}$ & $4: 1.3(0.98-1.68)$ & $4: 1.40(1.03-1.63)$ & $4: 1.20(1.03-1.70)$ \\
\hline S_crea & $0: 83.0(73.5-95.0)$ & $0: 80.0$ (69.3-93.3) & $0: 88.0(63.3-104)$ & $0: 62.0(57.3-104)$ \\
\hline \multirow[t]{4}{*}{$(\mu \mathrm{mol} / l)$} & $1: 83.0(79.5-93.0)$ & 1: $79.0(74.3-92.3)$ & $1: 85.0(66.8-106)$ & $1: 58.0(54.3-71.3)$ \\
\hline & $2: 82.0(74.5-89.0)$ & $2: 84.0(77.3-103)$ & $2: 84.0(76.8-104)$ & 2: $70.0(60.5-98.5)$ \\
\hline & $3: 80.0(69.5-86.0)$ & 3: 82.0 (68.8-98.8) & $3: 80.0(69.8-98.0)$ & 3: 64.0 (56.8-91.0) \\
\hline & $4: 75.5(71.5-84.0)$ & 4: $87.0(69.0-97.8)$ & 4: $80.0(64.8-109)$ & $4: 75.0(58.8-108)$ \\
\hline$S \_N G A L$ & $0: 126(91.5-239)^{3}$ & $0: 118(103-189)$ & $0: 139(132-224)$ & $0: 122(116-191)$ \\
\hline \multirow[t]{4}{*}{$(\mu g / l)$} & $1: 115(75.5-157)$ & 1: $106(69.8-155)$ & $1: 212(110-230)$ & 1: $127(66.5-129)$ \\
\hline & 2: $93.0(65.0-132)$ & $2: 130(78.0-180)$ & 2: $145(60.8-195)$ & 2: $105(81.8-129)$ \\
\hline & 3: $91.5(61.0-126)$ & 3: $90.0(55.0-159)$ & $3: 104(55.3-174)$ & $3: 121(42.0-197)$ \\
\hline & $4: 113(88.5-172)$ & 4: $120(68.3-190)$ & 4: $186(68.0-211)$ & $4: 130(100-188)$ \\
\hline
\end{tabular}

\footnotetext{
* statistically significantly higher than corresponding sample values from the Cycle I chemotherapy $(p<0.05) .{ }^{1}=$ statistically significantly higher than values in Sample 0 and Sample 4 of the same cycle $(p=0.03),{ }^{2}=$ statistically significantly lower than values in Sample 1 , Sample 2 and Sample 3 of the same cycle $(p=0.03),{ }^{3}=$ statistically significantly higher than values in Sample 2 and Sample 3 of the same cycle $(p=0.045)$
} 


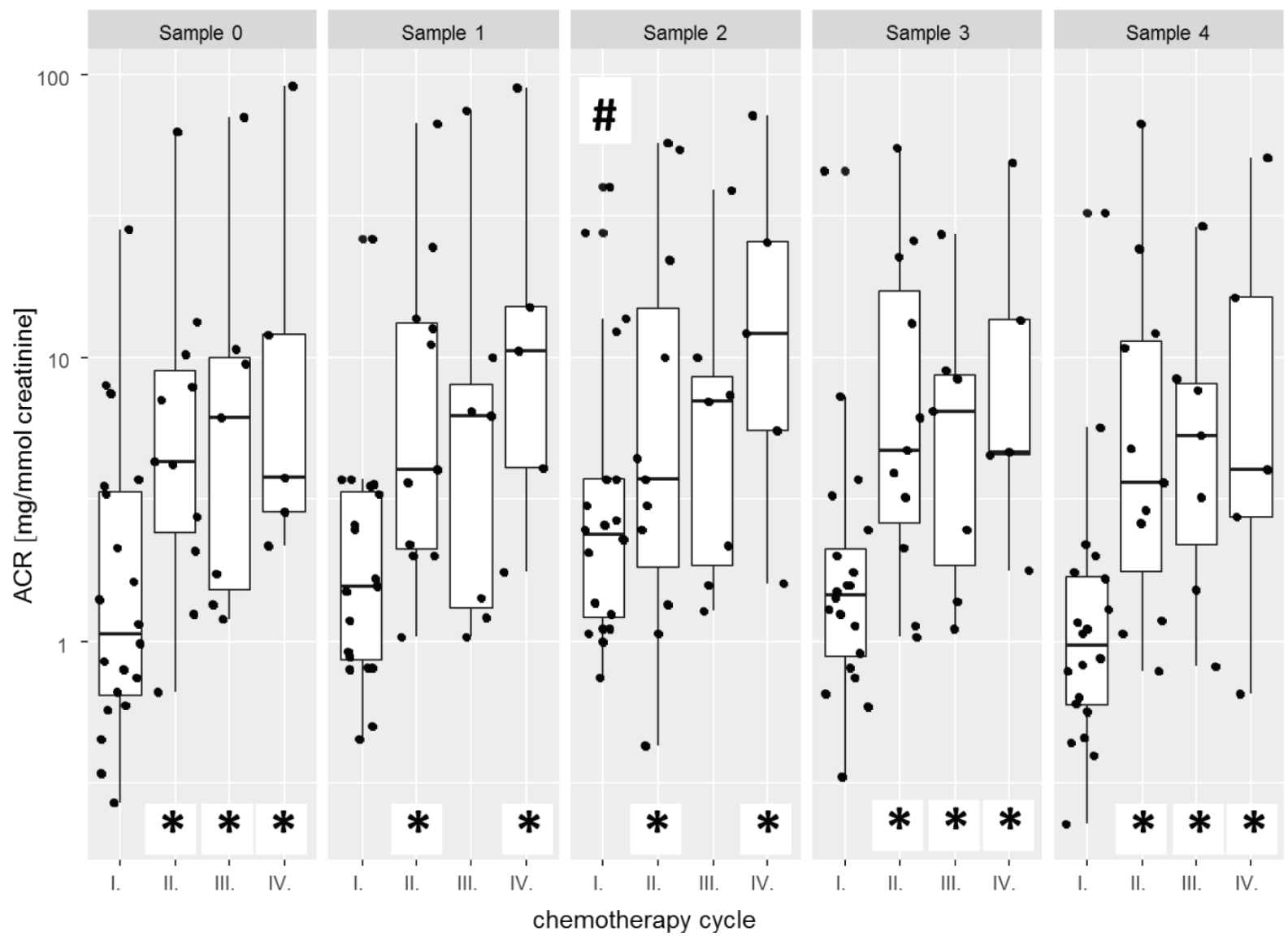

Fig. 1A. Boxplot showing changes in urine albumin/urine creatinine ratio (ACR) values between individual chemotherapy cycles (I to IV) in samples taken before (Sample 0), immediately after (Sample 1), in 3 hours (Sample 2), 6 hours (Sample 3) and 24 hours (Sample 4) after administering chemotherapy. Note the logarithmic scale on y axis. * Statistically significantly higher than corresponding sample values from the first chemotherapy cycle $(p<0.05)$. \# Statistically significantly higher than values in Sample 1, Sample 3 and Sample 4 of the same cycle $(p=0.03)$.

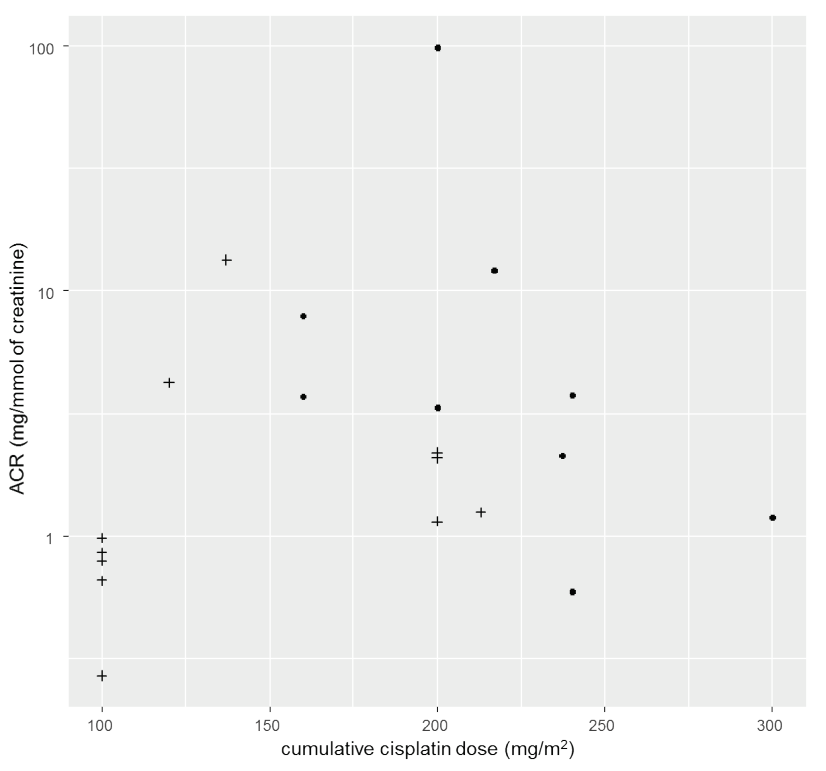

Fig. 1B. Correlation of ACR with cumulative dose of cisplatin $\left(\mathrm{mg} / \mathrm{m}^{2}\right)$ before the last applied cycle of chemotherapy (Sample 0). Crosses and full circles denote patients with and without clinical diagnosis of nephrotoxicity resp. Note logarithmic scale on y axis.
The univariate survival analysis for each marker with nephrotoxicity as a predicted variable showed that cystatin $\mathrm{C}$ appears to be a significant nephrotoxicity predictor of all measured markers $(p<0.05$, Table 2 , Fig. 4).

Correlation analysis revealed a robust correlation between measured value of urine NGAL and urine albumin $(\mathrm{r}=0.68, \mathrm{p}<0.0001)$. Interestingly, there was a statistically significant corrrelation (Table 3) of cumulative dose of cisplatin $\left(\mathrm{mg} / \mathrm{m}^{2}\right)$ in the last applied cycle of chemotherapy with ACR in Sample 0 in patients with clinical diagnosis of nephrotoxicity only $(r=0.67$, $\mathrm{p}=0.023$, Fig. 1B) and in Sample 2 in patients without clinical diagnosis of nephrotoxicity only $(r=-0.80$, $\mathrm{p}=0.009$ ). Furthemore, cumulative cisplatin dose significantly correlated with U_NGAL/U_crea in samples 2 and 3 in patients without clinical diagnosis of nephrotoxicity only $(\mathrm{r}=-0,69, \mathrm{p}=0.038$ and $\mathrm{r}=-0.835$, $\mathrm{p}=0.005$ resp.) and with eGFR estimated from serum cystatin $\mathrm{C}$ values in Sample $1(\mathrm{p}=0.46, \mathrm{p}=0.041)$. 
Table 3. Correlation of U_NGAL/U_crea, ACR and eGFR (cystatin C) with cumulative dose of cisplatin $\left(\mathrm{mg} / \mathrm{m}^{2}\right)$ in the last applied cycle of chemotherapy. Data are presented as correlation coefficient rho ( $p$-value) and correlations with $p$-value $<0.1$ are in bold. Correlation coefficients are calculated for all patients $(n=20)$, for patients with $(n=11)$ and without $(n=9)$ clinical diagnosis of toxicity and are provided separately for samples taken before (Sample 0), immediately after (Sample 1), in 3 hours (Sample 2), 6 hours (Sample 3) and 24 hours (Sample 4) after administering chemotherapy.

\begin{tabular}{|c|c|c|c|c|c|}
\hline & Sample 0 & Sample 1 & Sample 2 & Sample 3 & Sample 4 \\
\hline \multicolumn{6}{|l|}{ U_NGAL/U_crea } \\
\hline all & $0.34(0.14)$ & $0.15(0.53)$ & $0.05(0.81)$ & $-0.11(0.64)$ & $0.42(0.06)$ \\
\hline w nephrotoxicity & $0.48(0.13)$ & $-0.20(0.55)$ & $-0.16(0.63)$ & $-0.02(0.93)$ & $0.46(0.15)$ \\
\hline wo nephrotoxicity & $-0.65(0.058)$ & $-0.30(0.43)$ & $-0.69(0.038)$ & $-0.84(0.005)$ & $-0.23(0.55)$ \\
\hline \multicolumn{6}{|l|}{$A C R$} \\
\hline all & $0.26(0.26)$ & $0.26(0.28)$ & $0.07(0.76)$ & $-0.11(0.65)$ & $0.22(0.35)$ \\
\hline w nephrotoxicity & $0.67(0.023)$ & $0.33(0.32)$ & $0.03(0.92)$ & $0.34(0.31)$ & $0.10(0.77)$ \\
\hline wo nephrotoxicity & $-0.55(0.12)$ & $-0.24(0.53)$ & $-0.80(0.009)$ & $-0.60(0.09)$ & $-0.24(0.54)$ \\
\hline \multicolumn{6}{|l|}{ eGFR (cystatin C) } \\
\hline all & $0.43(0.056)$ & $0.46(0.041)$ & $0.29(0.22)$ & $0.23(0.34)$ & $0.18(0.44)$ \\
\hline w nephrotoxicity & $0.18(0.59)$ & $0.35(0.29)$ & $-0.17(0.61)$ & $-0.25(0.45)$ & $-0.24(0.47)$ \\
\hline wo nephrotoxicity & $0.025(0.95)$ & $0.32(0.40)$ & $0.06(0.86)$ & $0.20(0.60)$ & $0.17(0.66)$ \\
\hline
\end{tabular}

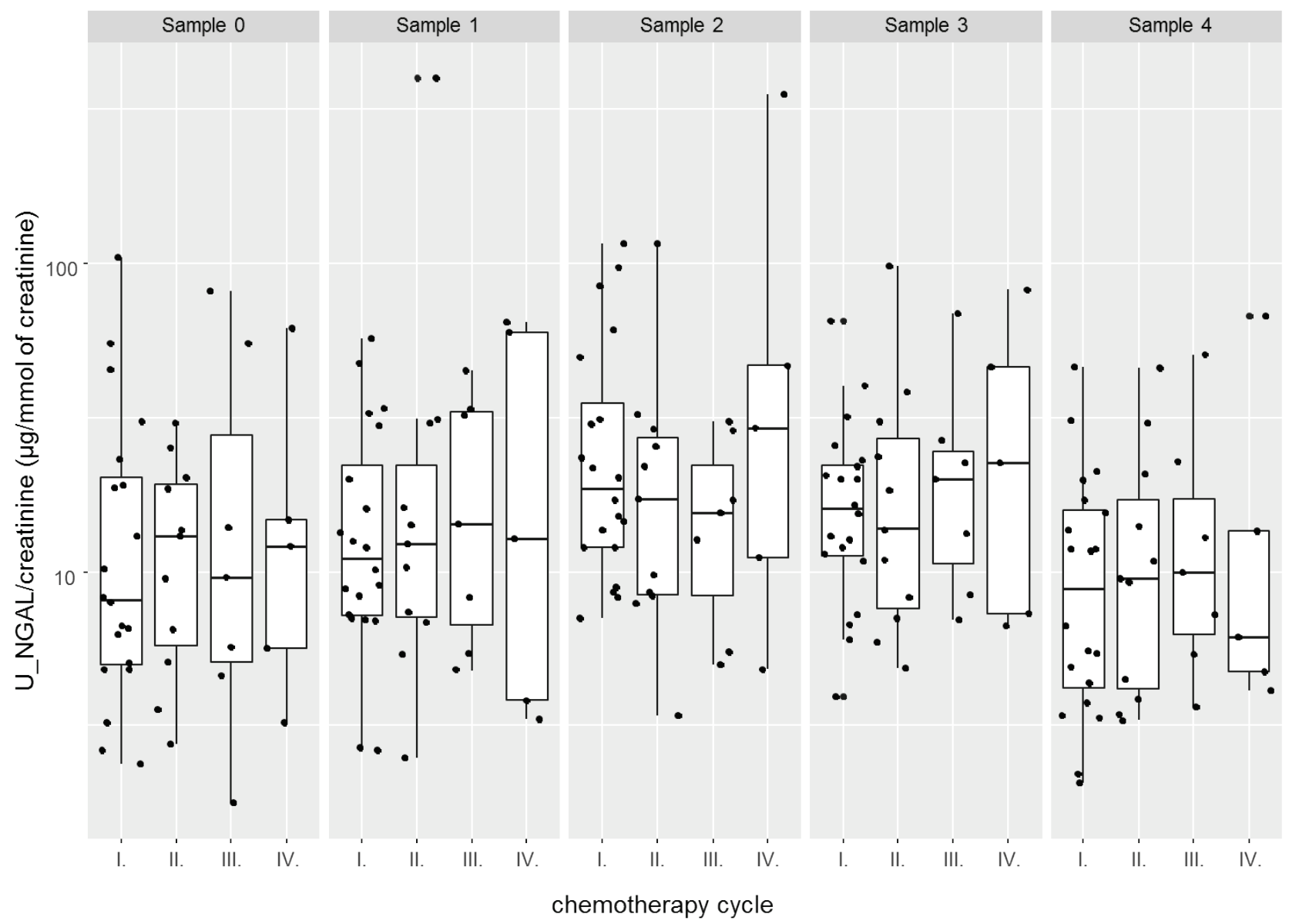

Fig. 2A. Boxplot showing changes in urine NGAL/urine creatinine ratio values between individual chemotherapy cycles (I to IV) in samples taken before (Sample 0), immediately after (Sample 1), in 3 hours (Sample 2), 6 hours (Sample 3) and 24 hours (Sample 4) after administering chemotherapy. Note the logarithmic scale on y axis. Values we not significantly changed neither within neither among chemotherapy cycles. 


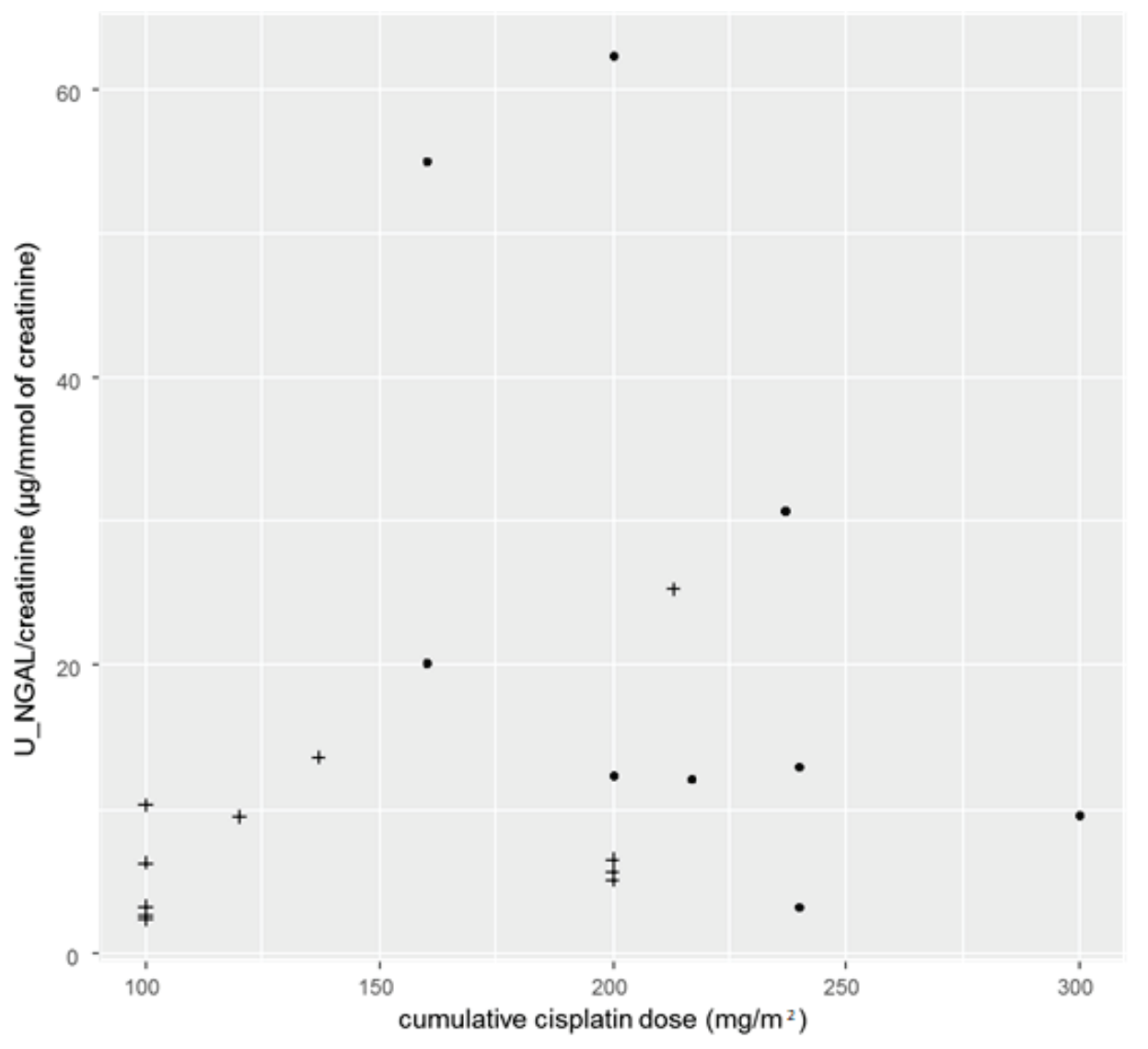

Fig. 2B. Correlation of urine NGAL/urine creatinine with cumulative dose of cisplatin $\left(\mathrm{mg} / \mathrm{m}^{2}\right)$ before the last applied cycle of chemotherapy (Sample 0 ). Crosses and full circles denote patients with and without clinical diagnosis of nephron-toxicity resp.

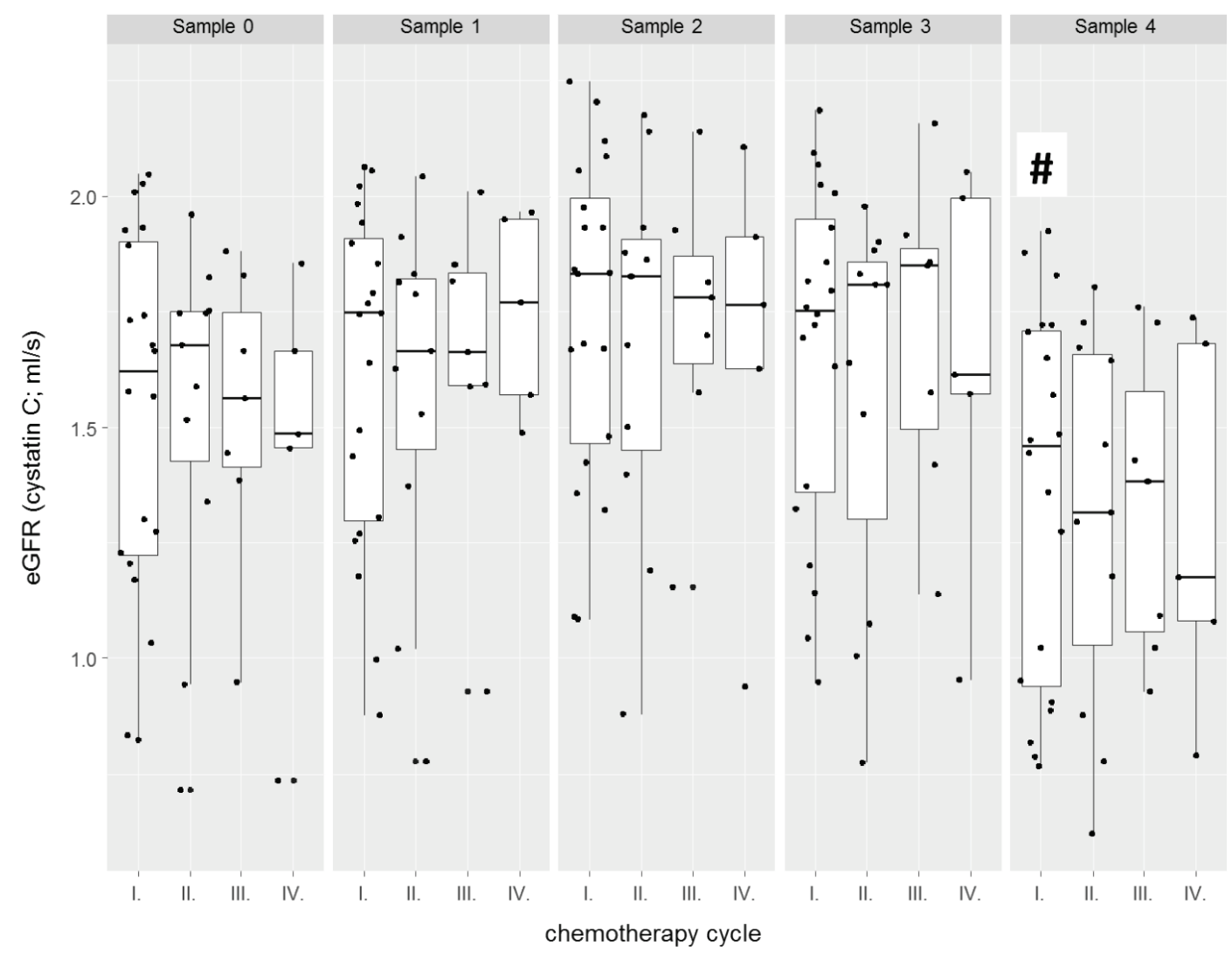

Fig. 3A. Boxplot showing changes in eGFR estimated by serum cystatin $C$ values between individual chemotherapy cycles (I to IV) in samples taken before (Sample 0), immediately after (Sample 1), in 3 hours (Sample 2), 6 hours (Sample 3) and 24 hours (Sample 4) after administering chemotherapy\# Statistically significantly lower than values in Sample 1, Sample 2 and Sample 3 of the same cycle $(p=0.03)$. 


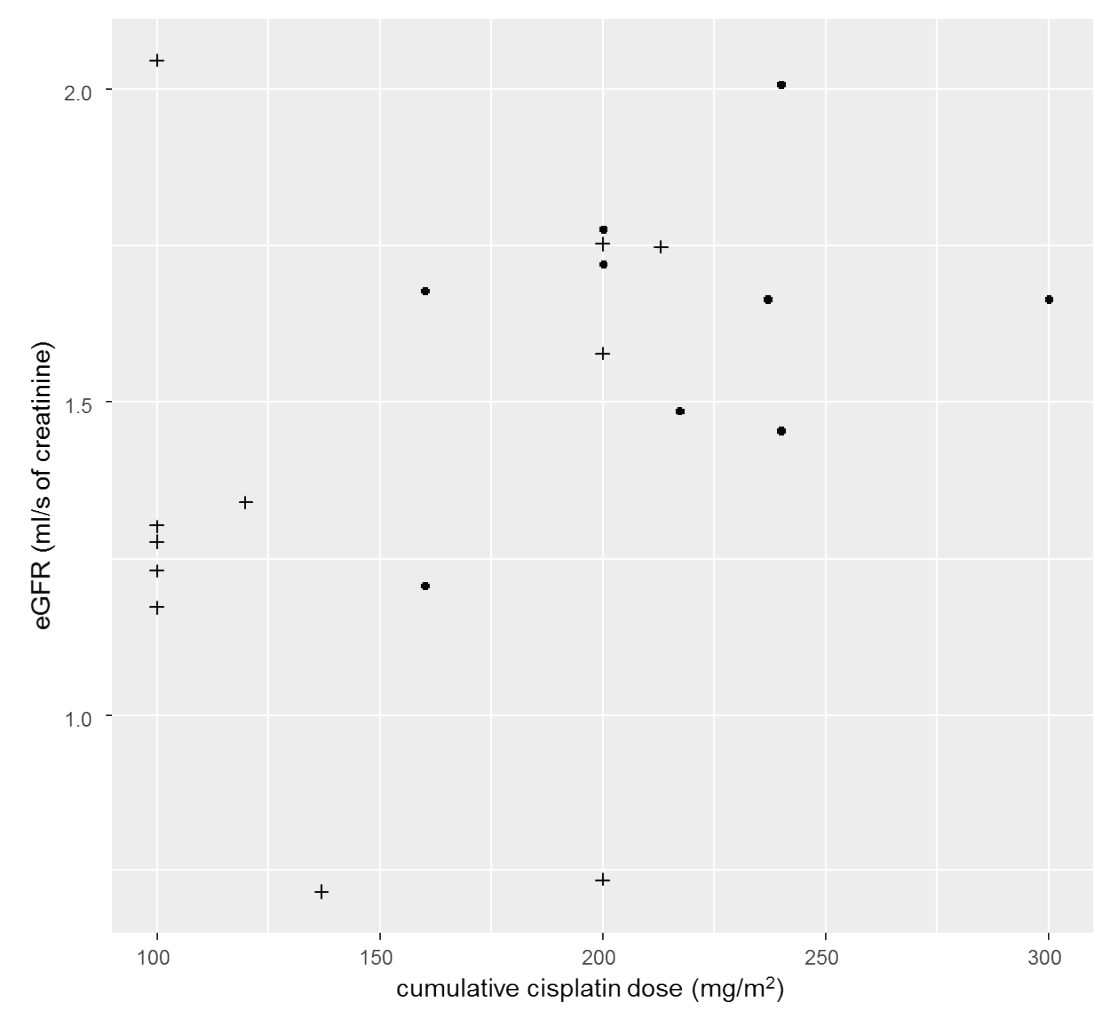

Fig. 3B. Correlation of eGFR estimated from serum cystatin $C$ values with cumulative dose of cisplatin $\left(\mathrm{mg} / \mathrm{m}^{2}\right)$ before the last applied cycle of chemotherapy (Sample 0 ). Crosses and full circles denote patients with and without clinical diagnosis of nephrotoxicity resp.

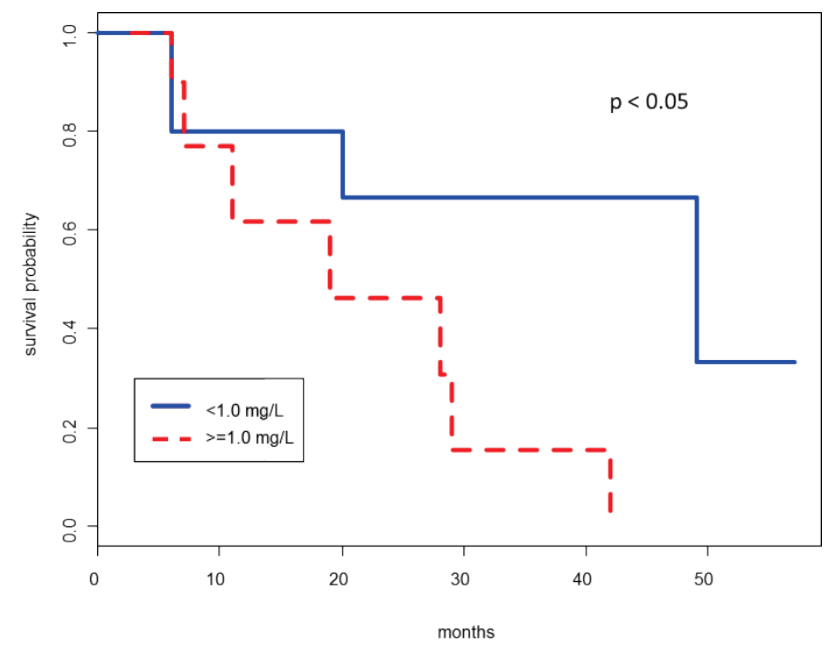

Fig. 4. Univariate survival analysis with nephrotoxicity as a predicted variable and cystatin $\mathrm{C}$ as a predictor.

\section{Discussion}

In our study, we compared changes in biomarkers among individual samples after administering cisplatin (a short-time view of cisplatin toxicity) and among chemotherapy cycles (a long-time view of cumulative effects of cisplatin) in oncological patients. We measured 5 markers: serum and urine NGAL, serum creatinine and cystatin $\mathrm{C}$ and urine albumin. The comparison of their clinical feasibility was derived from changes after cisplatin administration. Although there were no conspicuous changes of measured markers in a short period after cisplatin administration, in the longterm period, urinary albumin increase was consistently detected in oncologic patients undergoing chemotherapy containing cisplatin. Our main finding is that urinary albumin, in direct comparison with urinary NGAL, more consistently increases after chemotherapy containing cisplatin (Figs 1A, 2A). The design of our study follows common clinical practice of cumulative administration of cisplatin in chemotherapy cycles. On the contrary, most of the published results deal with time-dependent changes after single cisplatin dose.

Concentrating on short-time view of cisplatin toxicity, we detected significant increase of ACR and decrease of eGFR up to 24 hours after cisplatin administration in the first chemotherapy cycle only. Unfortunately, these changes were not statistically significant in subsequent chemotherapy cycles. The most probable explanation of this observation is the significantly decreased statistical power caused by reduction of participants in chemotherapy cycles II and especially III and IV. Different designs of published studies make it difficult to compare results with our study. Nevertheless, in 33 cisplatin treated oncologic patients Lin et al. (2013) found a significant increase of ACR 6 and 96 hours and an increase of urinary NGAL between 12 and 72 hours after cisplatin infusion in patients with subsequent AKI only. In Lin's study, 
10 patients $(30 \%)$ of patients have greater than $25 \%$ decrease of eGFR, whereas in our study, just 1 patient (5\%) achieved this limit. Moreover, the clinical approach to diagnosis of AKI in clinical practice is different in different clinical contexts (e.g. in sepsis; Chvojka et al. 2010) and cannot be easily translated from one context to another. In comparison with Lin's study, we also found an increase of ACR but we didn't detect a significant increase of urinary NGAL. Similarly, Gaspari observed a significant increase of urinary NGAL 1, 2 and 3 days after cisplatin administration in 12 patients with AKI only (Gaspari et al. 2010). Although there was an obvious increasing trend for urinary NGAL in our study, heterogeneity of responses to cisplatin treatment prevented it from being significant. Similar observation was found by George (George et al. 2017), who analyzed samples from 57 patients 3 and 10 days after cisplatin treatment: there were no changes in urinary NGAL levels but urinary albumin increased.

Uniqueness of our results lies in the description of long-term view on changes in measured biomarkers after repetitive cisplatin administrations in the setting of real-world chemotherapy cycles. We are not aware of any similar published studies. According to our results, ACR has a consistent and statistically significant pattern of increase with increasing number of cisplatin containing chemotherapy cycles (Fig. 1A). On contrary, response of urinary NGAL to increasing number of cisplatin containing chemotherapy cycles is more heterogenous and thus not statistically significant (Fig. 2A). We did not find any significant long-term effect of increasing number of cisplatin containing chemotherapy cycles on estimated glomerular filtration rate. However, correlation of cumulative dose of cisplatin with measured markers doesn't provide any robust correlation that is consistent across all sampling intervals (Table 3 ). The failure to provide an evidence of cumulative dose-dependent relationship to urinary NGAL or ACR can be caused by gradual selection of patients that are less sensitive to nephrotoxic effects of cisplatin. These patients have low levels of urinary NGAL and ACR despite of high cumulative dose of cisplatin (Fig. 1B, 2B). We can only hypothesize that paradoxical positive correlation of cumulative cisplatin dose and eGFR estimated from serum cystatin $\mathrm{C}$ before and after administration of the last chemotherapy (Fig. 3 B, Table 1II) reflects a decrease of cystatin $\mathrm{C}$ production due to decrease of tumor mass during the treatment. In the literature, some authors prove decrease of eGFR, e.g. De Jongh et al. ascertained that serum creatinine increased over upper reference limit in $41 \%$ of patients after treatment with cisplatin (de Jongh et al. 2003) but serum cystatin C was not assessed in this study. As mentioned above, the main reason of failing to prove significant long-term effects on urinary NGAL and eGFR in our study can be the small numbers of participants in chemotherapy cycles II, III and IV. That is why our results should be interpreted with caution. Moreover, as mentioned in the Introduction, both serum creatinine and serum cystatin $\mathrm{C}$ as markers of GFR can have significant drawbacks in oncologic patients and studies with precise and non-biased measurement of glomerular filtration rate are needed.

Heterogeneity of results found in literature can be partly explained by the fact that absolute concentration of urinary markers is severely influenced by large amounts of hydration (e.g. 5 litres of i.v. fluids a day) used in cisplatin dosage protocols. Urine marker correction to urine creatinine does not have to be optimum in oncological patients because creatinine excretion can be substantially influenced by nutrition and catabolic state and subsequent correction of marker levels to urine creatinine concentration might distort information (Drott et al. 1988; Waikar et al. 2010). Time urine collection would probably be a better solution, but current clinical experience with a very high frequency of errors in urine collection invalidates this solution too. Another factor which may decrease urine NGAL validity in oncological patients is the presence of tumor itself and possible NGAL expression in tumor tissue and many other tissues. Increased NGAL production was described e. g. in esophageal, lung, as well as colon tumors (Chakraborty et al. 2012).

In conclusion, ACR was the only measured marker that consistently increased with increased number of cisplatin containing chemotherapy cycles in oncologic patients.

\section{Conflict of Interest}

There is no conflict of interest.

\section{Acknowledgements}

This study was supported by the grant of Ministry of Health of the Czech Republic - Conceptual Development of Research Organization (Faculty Hospital in Pilsen FNP1, 00669806). The study was supported by project No. CZ.02.1.01/0.0/0.0/16_019/0000787 „Fighting INfectious Diseases“, awarded by the MEYS CR, financed from EFRR. 


\section{References}

CHAKRABORTY S, KAUR S, GUHA S, BATRA SK: The multifaceted roles of neutrophil gelatinase associated lipocalin (NGAL) in inflammation and cancer. Biochim Biophys Acta 1826: 129-169, 2012. https://doi.org/10.1016/j.bbcan.2012.03.008

DE JONGH FE, VAN VEEN RN, VELTMAN SJ, DE WIT R, VAN DER BURG MEL, VAN DEN BENT MJ, PLANTING AST, GRAVELAND WJ, STOTER G, VERWEIJ J: Weekly high-dose cisplatin is a feasible treatment option: analysis on prognostic factors for toxicity in 400 patients. Br J Cancer 88: 1199-1206, 2003. https://doi.org/10.1038/sj.bjc.6600884

DEVARAJAN P: Neutrophil gelatinase-associated lipocalin: a promising biomarker for human acute kidney injury. Biomark Med 4: 265-280, 2010. https://doi.org/10.2217/bmm.10.12

DROTT C, SVANINGER G, LUNDHOLM K: Increased urinary excretion of cortisol and catecholami-NES in malnourished cancer patients. Ann Surg 208: 645-650, 1988. https://doi.org/10.1097/00000658-198811000$\underline{00017}$

GASPARI F, CRAVEDI P, MANDALÀ M, PERICO N, LEON FR DE, STUCCHI N, FERRARI S, LABIANCA R, REMUZZI G, RUGGENENTI P: Predicting cisplatin-induced acute kidney injury by urinary neutrophil gelatinase-associated lipocalin excretion: a pilot prospective case-control study. Nephron Clin Pract 115: c154-c160, 2010. https://doi.org/10.1159/000312879

GAYGISIZ Ü, AYDOGDU M, BADOGLU M, BOYACI N, GÜLLÜ Z, GÜRSEL G: Can admission serum cystatin C level be an early marker subclinical acute kidney injury in critical care patients? Scand. J Clin Lab Invest 76 : 143-150, 2016. https://doi.org/10.3109/00365513.2015.1126854

GEORGE B, WEN X, MERCKE N, GOMEZ M, O'BRYANT C, BOWLES DW, HU Y, HOGAN SL, JOY MS, ALEKSUNES LM: Profiling of kidney injury biomarkers in patients receiving cisplatin: Time-dependent changes in the absence of clinical nephrotoxicity. Clin Pharmacol Ther 101: 510-518, 2017. https://doi.org/10.1002/cpt.606

HAASE M, DEVARAJAN P, HAASE-FIELITZ A, BELLOMO R, CRUZ DN, WAGENER G, KRAWCZESKI CD, KOYNER JL, MURRAY P, ZAPPITELLI M, GOLDSTEIN SL, MAKRIS K, RONCO C, MARTENSSON J, MARTLING C-R, VENGE P, SIEW E, WARE LB, IKIZLER A, MERTENS PR: the outcome of neutrophil gelatinase-associated lipocalin (NGAL)-positive subclinical acute kidney injury: a multicenter pooled analysis of prospective studies. J Am Coll Cardiol 57: 1752-1761, 2011. https://doi.org/10.1016/j.jacc.2010.11.051

HAASE-FIELITZ A, HAASE M, DEVARAJAN P: Neutrophil gelatinase-associated lipocalin as a biomarker of acute kidney injury: a critical evaluation of current status. Ann Clin Biochem 51: 335-351, 2014. https://doi.org/10.1177/0004563214521795

HEERSPINK HJL, GANSEVOORT RT: Albuminuria Is an appropriate therapeutic target in patients with CKD: The pro view. Clin J Am Soc Nephrol 10: 1079-88, 2015. https://doi.org/10.2215/CJN.11511114

KIDNEY DISEASE: IMPROVING GLOBAL OUTCOMES (KDIGO) CKD WORK GROUP: KDIGO 2012 CLINICAL PRACTICE GUIDELINE FOR THE EVALUATION AND MANAGEMENT OF CHRONIC KIDNEY DISEASE. Kidney Int 3: 1-150, 2013.

KIM J: Poly(ADP-ribose) polymerase activation induces high mobility group box 1 release from proximal tubular cells during cisplatin nephrotoxicity. Physiol Res 65: 333-340, 2016. https://doi.org/10.33549/physiolres.932948

KOS J, S̆TABUC B, CIMERMAN N, BRÜNNER N: Serum Cystatin C, a new marker of glomerular filtration rate, is increased during malignant progression. Clin Chem 44: 2556-2557, 1998. https://doi.org/10.1093/clinchem/44.12.2556

LIN HY-H, LEE S-C, LIN S-F, HSIAO H-H, LIU Y-C, YANG W-C, HWANG D-Y, HUNG C-C, CHEN H-C, GUH J-Y: Urinary neutrophil gelatinase-associated lipocalin levels predict cisplatin-induced acute kidney injury better than albuminuria or urinary cystatin C levels. Kaohsiung J Med Sci 29: 304-311, 2013. https://doi.org/10.1016/j.kjms.2012.10.004 
MAGHSOUDI O, MIRJALILI SH, DOLATABADI M, JOSHAGHANI MF, ZAREA M, YAHAGHI E, MOKARIZADEH A: Investigations of renal function using the level of neutrophil gelatinase-associated lipocalin associated with single-dose of cisplatin during chemotherapy. Diagn Pathol 10: 98, 2015. https://doi.org/10.1186/s13000-015-0338-5

MILLER RP, TADAGAVADI RK, RAMESH G, REEVES WB: Mechanisms of Cisplatin Nephrotoxicity. Toxins 2: 2490-2518, 2010. https://doi.org/10.3390/toxins2112490

MISHRA J, MA Q, PRADA A, MITSNEFES M, ZAHEDI K, YANG J, BARASCH J, DEVARAJAN P: Identification of neutrophil gelatinase-associated lipocalin as a novel early urinary biomarker for ischemic renal injury. $\mathrm{J}$ Am Soc Nephrol 14: 2534-2543, 2003. https://doi.org/10.1097/01.ASN.0000088027.54400.C6

NICKOLAS TL, BARASCH J, DEVARAJAN P: Biomarkers in acute and chronic kidney disease. Curr Opin Nephrol Hypertens 17: 127-132, 2008. https://doi.org/10.1097/MNH.0b013e3282f4e525

TSIGOU E, PSALLIDA V, DEMPONERAS C, BOUTZOUKA E, BALTOPOULOS G: Role of new biomarkers: functional and structural damage. Crit Care Res Pract, 2013. https://doi.org/10.1155/2013/361078

WAIKAR SS, SABBISETTI VS, BONVENTRE JV: Normalization of urinary biomarkers to creatinine during changes in glomerular filtration rate. Kidney Int 78: 486-494, 2010. https://doi.org/10.1038/ki.2010.165

XU Y, DING Y, LI X, WU X: Cystatin C is a disease-associated protein subject to multiple regulation. Immunol Cell Biol 93: 442-451, 2015. https://doi.org/10.1038/icb.2014.121 